\title{
Orthorexia Nervosa in College Students: Eating Disorder History, Gender, and Dieting Behaviors
}

Katrina Agger, Brittany Moran, and Zhiping $Y u, P h D, R D N, L D N$

Department of Nutrition and Dietetics University of North Florida, Jacksonville, FL 32224
UNIVERSITY of NORTH FLORIDA

\section{N T R O D U C T I O N}

Orthorexia nervosa (ON) is the term used to describe the persistent preoccupation with eating healthy, first coined in 1997. ${ }^{1}$ College students are a high-risk group for the development of $\mathrm{ON}$ tendencies, with prevalence rates of $\mathrm{ON}$ in college students estimated at $25.2 \% .^{2}$ This may be because young people starting university experience greater social pressure and thus develop unrealistic expectations for themselves, leading them to adopt compensatory behaviors such as disordered eating patterns and/or dieting for weight loss. ${ }^{3}$ Female students may also feel greater pressure to conform to a gender-specific "thin ideal," and a fixation on healthy eating as seen in ON may be used by some as a socially acceptable method of expressing full-syndrome disordered eating behaviors as seen in anorexia nervosa and other eating disorders (ED). ${ }^{4}$

Currently, there is no substantial research on how eating disorder history and disordered dieting behaviors affect $\mathrm{ON}$ in this population. There have been mixed conclusions on whether $\mathrm{ON}$ is seen in higher rates in male or female college students.

\section{O B J E C TIVES}

This study aimed to assess how the factors of eating disorder history, gender, and dieting behaviors affect $\mathrm{ON}$ in college students at the University of North Florida.

\section{RESEARCH HYPOTHESES}

- Students with a history of eating disorder(s) will demonstrate a higher prevalence of ON compared to students without a history of eating disorder(s).

- Female students will have a higher prevalence of ON compared to male students.

- College students who score higher disordered dieting behavior scores will demonstrate a higher prevalence of $\mathrm{ON}$.

\section{METHODS}

Students 18 years or older at the University of North Florida enrolled in summer 2018 were invited to take an online survey distributed via Qualtrics' email system. Survey questions included those from the Bratman Orthorexia Test (BOT), the Eating Attitude Test-26 (EAT-26), and questions regarding participant characteristics.

- EAT-26: is a widely used screening tool used to assess eating disorder risk based on three subscales: dieting, food preoccupation, and oral control. 6

- BOT: is a 10 -item screening tool developed by Steven Bratman with yes/no answers to assess food attitudes and ON tendencies in US populations.

- Participant characteristic questions included age, gender, history of eating disorder, race, height, and weight. All data analysis were performed using SPSS.

\section{RES U L T S}

Table 2: ON/ED Risk in College Students by History of Eating Disorder

\begin{tabular}{|c|c|c|c|c|c|}
\hline & With ED history ( $n=59$ ) & \begin{tabular}{|l|} 
Without ED \\
history ( $\mathrm{n}=352)$
\end{tabular} & Total & $\mathbf{F}$ & P-value \\
\hline EAT Total Score & $20.2 \pm 13.2$ & $9.7 \pm 9.1$ & $11.2 \pm 10.4$ & 58.1 & $<0.0001$ \\
\hline ON Total Score & $5.6 \pm 2.3$ & $4.3 \pm 2.4$ & $4.4 \pm 2.4$ & 15.1 & $<0.0001$ \\
\hline D Risk & & & & 44.1 & $<0.0001$ \\
\hline EAT $\geq 20(\%)$ & $29(49.2)$ & $46(13.1)$ & $75(18.2)$ & & \\
\hline EAT <20 (\%) & $30(50.8)$ & $306(86.9)$ & $336(81.8)$ & & \\
\hline ON Risk & & & & 7.7 & 0.006 \\
\hline $\mathrm{ON}=5-10(\%)$ & $39(66.1)$ & $164(46.6)$ & $203(49.4)$ & & \\
\hline ON $<5(\%)$ & 20(33.9) & $188(53.4)$ & $208(50.6)$ & & \\
\hline D+ON Risk & & & & 35.4 & $<0.0001$ \\
\hline$E A T \geq 20 \& O N=5-10$ & $25(42.4)$ & $41(11.6)$ & $66(16.1)$ & & \\
\hline All others & $34(57.6)$ & $311(88.4)$ & $345(83.9)$ & & \\
\hline
\end{tabular}

Comparing to students without ED history, students with ED history had higher ON total score 5.6 vs. $4.3, p<0.0001)$ and higher percentage being a health fanatic or orthorexic $(66.1 \%$ vs. $46.6 \%, p=0.006)$.

\begin{tabular}{|c|c|c|c|c|c|}
\hline & Female $(n=307)$ & Male ( $n=101)$ & Total & $\mathbf{F}$ & $\mid \begin{array}{l}\mathrm{P} \text { - } \\
\text { value }\end{array}$ \\
\hline EAT Total Score & $12.1 \pm 11.0$ & $8.7 \pm 7.7$ & $11.2 \pm 10.4$ & 7.97 & 0.005 \\
\hline ON Total Score & $4.6 \pm 2.4$ & $4.0 \pm 2.3$ & $4.4 \pm 2.4$ & 5.42 & 0.02 \\
\hline ED Risk & & & & 4.75 & 0.03 \\
\hline$E A T \geq 20(\%)$ & $63(20.5)$ & $11(10.9)$ & 74(18.1) & & \\
\hline EAT $<20(\%)$ & $244(79.5)$ & $90(89.1)$ & $334(81.9)$ & & \\
\hline ON Risk & & & & 3.37 & 0.07 \\
\hline ON=5-10(\%) & $160(52.1)$ & $42(41.6)$ & $202(49.5)$ & & \\
\hline ON $<5(\%)$ & $147(47.9)$ & $59(58.4)$ & $206(50.5)$ & & \\
\hline ED+ON Risk & & & & & \\
\hline EAT $\geq 20 \& O N=5-10$ & $57(18.6)$ & $9(8.9)$ & $16.2(66)$ & 5.23 & 0.02 \\
\hline All others & $250(81.4)$ & 92(91.1) & $83.8(342)$ & & \\
\hline
\end{tabular}

Female students had higher $\mathrm{ON}$ total scores compared to male students( 4.6 vs. 4.0

$\mathrm{p}=0.02$ ) but statistically the same percentage of females had high ON risk as males $(52.1 \%$ vs. $41.6 \%, p=0.07$.

Table 4: Association between Bratman Orthorexia Test (BOT) and Subscales of the Eating

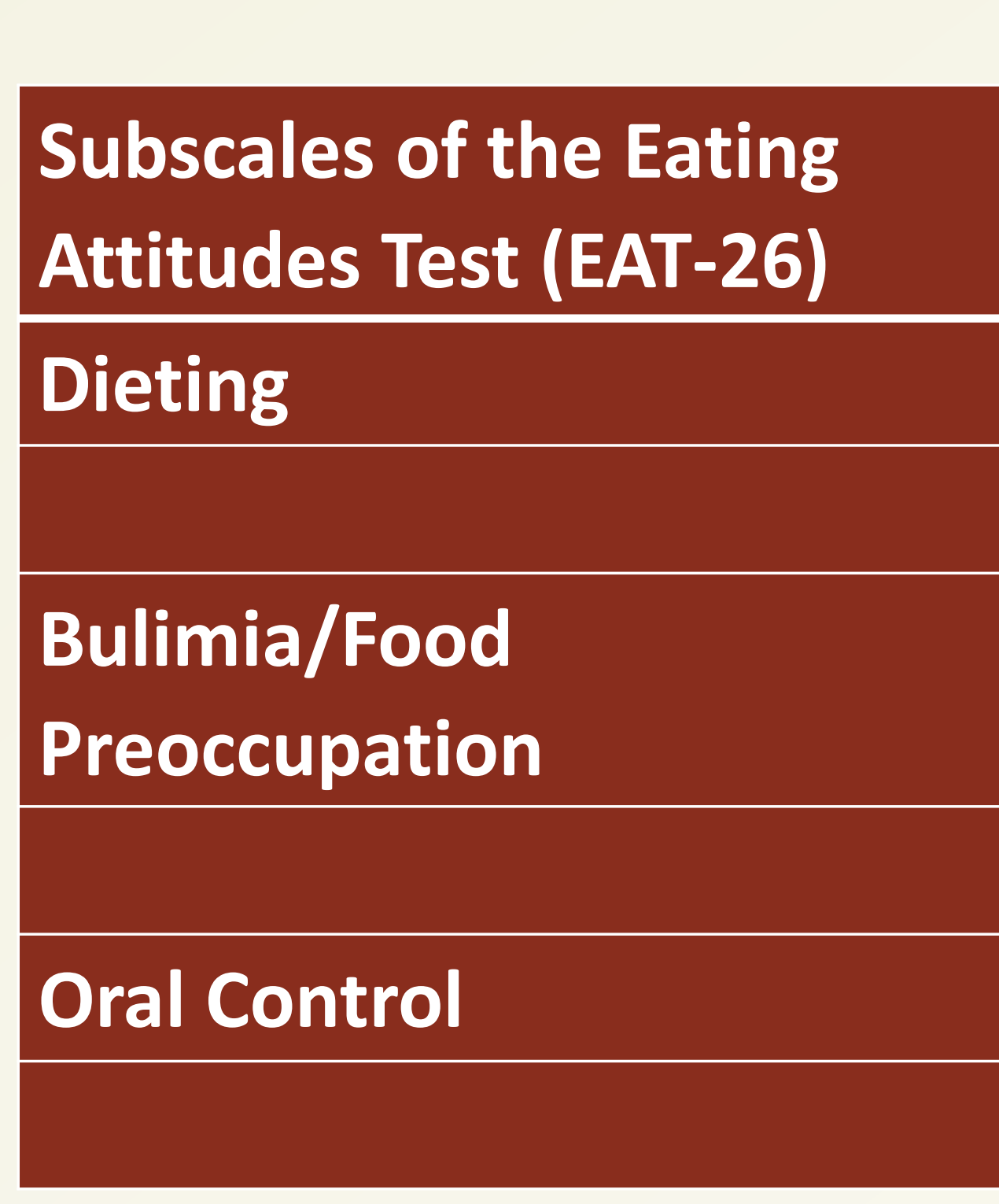
Attitudes Test (EAT-26 in College Students

\begin{tabular}{|l|l|l|l|l|l|}
\hline BOT Score & N & Mean & SD & F & P-value \\
\hline ON (5-10) & 203 & 11.7 & 8.1 & 158.0 & $<0.0001$ \\
\hline ON (<5) & 208 & 3.7 & 4.3 & & \\
ON (5-10) & 203 & 2.4 & 3.2 & 63.6 & $<0.0001$ \\
& & & & & \\
ON (<5) & 208 & 0.5 & 1.2 & & \\
ON (5-10) & 203 & 2.3 & 2.7 & 1.5 & 0.23 \\
ON (<5) & 208 & 2.0 & 2.5 & & \\
\hline
\end{tabular}

Students who are characterized as health fanatic or orthorexic had higher dieting scores comparing to those who are not (11.7 vs. $3.7, p<0.0001)$
Table 1: Demographic Profile of Participants

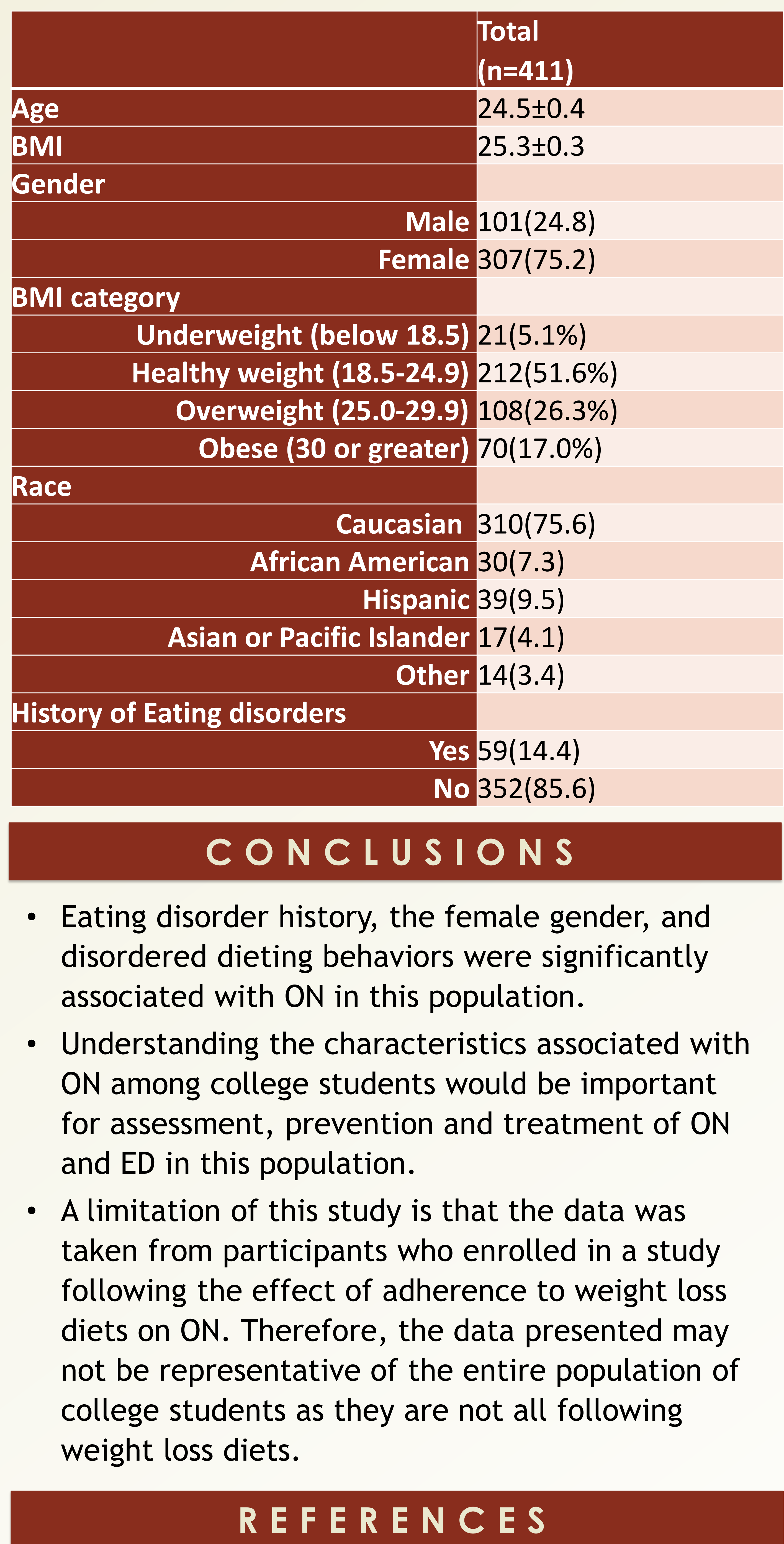

1. Oberle CD, Samaghabadi RO, Hughes EM. Appetite. 2017;108:30310. doi:10.1016/j.appet.2016.10.02

2. Parra-Fernández ML, Onieva-Zafra MD, Fernández-Martínez E, Abreu-Sánchez A, Fernández-Muñoz JJ, international Journal of Environmental Research and Public Health. 2019:16(14):2459. doi:10.3390/ijerph16142459

. Plichta M. Jezewska-Zychowicz M. Appetite. 2019;137:114-123. doi:10.1016/j.appet.2019.02.022

4. Brytek-Matera A, Rogoza R, Gramaglia C, Zeppegno P. BMC 5. Garner DM, Olmsted MP, Bohr Y, Ga

6. Bundros J, Clifford D, Silliman K, Neyman Morris M. Appetite. 2016;101:86-94. doi:10.1016/j.appet.2016.02.144 